\title{
Effects of Ketamine on Context-Processing Performance in Monkeys: A New Animal Model of Cognitive Deficits in Schizophrenia
}

\author{
Rachael K Blackman ${ }^{1,2,3}$, Angus W MacDonald III, ${ }^{4,5}$ and Matthew V Chafee, ${ }^{*, 2,3,5}$ \\ 'Medical Scientist Training Program (MD/PhD), University of Minnesota, Minneapolis, MN, USA; ${ }^{2}$ Department of Neuroscience, University of \\ Minnesota, Minneapolis, MN, USA; ${ }^{3}$ Brain Sciences Center, VA Medical Center, Minneapolis, MN, USA; ${ }^{4}$ Department of Psychology, University of \\ Minnesota, Minneapolis, MN, USA; ${ }^{5}$ Center for Cognitive Sciences, University of Minnesota, Minneapolis, MN, USA
}

\begin{abstract}
Cognitive deficits are at the crux of why many schizophrenia patients have poor functional outcomes. One of the cognitive symptoms experienced by schizophrenia patients is a deficit in context processing, the ability to use contextual information stored in working memory to adaptively respond to subsequent stimuli. As such, context processing can be thought of as the intersection between working memory and executive control. Although deficits in context processing have been extensively characterized by neuropsychological testing in schizophrenia patients, they have never been effectively translated to an animal model of the disease. To bridge that gap, we trained monkeys to perform the same dot pattern expectancy (DPX) task, which has been used to measure context-processing deficits in human patients with schizophrenia. In the DPX task, the first stimulus in each trial provides the contextual information that subjects must remember in order to appropriately respond to the second stimulus in the trial. We found that administration of ketamine, an $\mathrm{N}$-methyl-D-aspartate receptor antagonist, in monkeys caused a dose-dependent failure in context processing, replicating in monkeys the same specific pattern of errors committed by patients with schizophrenia when performing the same task. Therefore, our results provide the first evidence that context-processing dysfunction can be modeled in animals. Replicating a schizophrenia-like behavioral performance pattern in monkeys performing the same task used in humans provides a strong bridge to better understand the biological basis for this psychiatric disease and its cognitive manifestations using animal models.
\end{abstract}

Neuropsychopharmacology (2013) 38, 2090-2100; doi:10.1038/npp.2013.1 I8; published online 5 June 2013

Keywords: context processing; monkey; executive control; NMDA antagonist; schizophrenia; translational

\section{INTRODUCTION}

Schizophrenia is characterized by positive, negative, and cognitive symptoms. Cognitive dysfunction is an aspect of the disease that is responsible for a substantial fraction of the disease burden associated with schizophrenia and has been shown to predict functional outcome (Green et al, 2000). However, it is an aspect of the disease that is not improved substantially by typical neuroleptic medications and is not improved consistently by atypical neuroleptics (Mortimer, 1997; Stip et al, 2005). Therefore, there is a need for a better understanding of the underlying changes in neuronal function at a cellular and synaptic level in schizophrenia, which are responsible for cognitive deficits in the disease, as this knowledge is likely to enhance our

* Correspondence: Professor MV Chafee, Brain Sciences Center (I I B), VA Medical Center, I Veterans Drive, Minneapolis, MN 55417, USA, Tel: + I 612467 3542, Fax: + I 6124672283 ,

E-mail: chafe00।@umn.edu

Received 25 January 2013; revised 7 April 2013; accepted 15 April 2013; accepted article preview online 10 May 2013 ability to identify more effective therapies to restore normal cognitive function. Animal models of schizophrenia can have an important role in this effort, as they provide a means to test hypotheses about the relation between altered neural and cognitive function in the disease state. They may be used to help elucidate the neural mechanisms by which the disease and its associated symptoms arise.

Here we examine in monkeys one aspect of cognitive processing that has not been previously studied in animal models but is frequently deficient in schizophrenia patients: context processing. Context processing is the ability to use contextual information encoded and maintained in working memory to flexibly modify responses to later stimuli (Barch et al, 2003; MacDonald, 2008). The construct of context processing (Cohen and Servan-Schreiber, 1992; MacDonald, 2008) incorporates and attempts to unify several observations of behavioral deficits in schizophrenia. These include not only a basic defect in the ability to store information in working memory (Castner et al, 2004), but also additional deficits in selective attention (Nuechterlein and Dawson, 1984), response inhibition (Abramczyk et al, 1983), and executive control (Sullivan et al, 1993) that have been 
described in the disease. Context-processing deficits in schizophrenia reflect the inability to use information stored in working memory to implement computational flexibility (Cohen and Servan-Schreiber, 1992; Goodwin et al, 2012; MacDonald, 2008; Miller and Cohen, 2001). Operationally, context processing is defined as the ability to produce different responses to the same sensory input as a function of stored contextual information, such as goals or rules (Miller and Cohen, 2001; Servan-Schreiber et al, 1996). The coupling of working memory and executive control components in the construct of context processing has proven to provide a sensitive measure of cognitive dysfunction in schizophrenia. For example, context-processing deficits are found in both in medicated and unmedicated first-episode schizophrenia patients (Servan-Schreiber et al, 1996) as well as in their first-degree relatives (MacDonald et al, 2003). In addition, deficits in context processing significantly correlate with deficits in the Stroop task (Cohen et al, 1999), supporting the construct validity of context-processing tasks as measures of cognitive operations that extend beyond working memory to include response inhibition and executive control (MacDonald, 2008). Further, patients with schizophrenia are impaired on context-processing tasks when there is no delay between stimuli within the trial (MacDonald et al, 2003), suggesting that context-processing deficits cannot be attributed solely to a failure of working memory maintenance. Contextprocessing dysfunction on the AX-continuous performance task (AX-CPT) appears to be specific to schizophrenia spectrum disorders including schizophrenia and schizotypal personality disorder (Barch et al, 2004) compared with non-schizophrenia psychotic diseases, bipolar disorder, and depression (Supplementary Discussion; Barch et al, 2003; Brambilla et al, 2007; Holmes et al, 2005; MacDonald et al, 2005a).

In this study, we trained monkeys to perform the dot pattern expectancy (DPX) task. This task has been validated as an effective measure of context-processing deficits in schizophrenia patients (Henderson et al, 2012; Jones et al, 2010; Lopez-Garcia et al, 2012; MacDonald et al, 2005b; Zhang et al, 2012). The DPX task is a variant of the AX-CPT that has been extensively used in prior studies of cognitive dysfunction in schizophrenia (Barch et al, 2003; Chung et al, 2011; Cohen et al, 1999; MacDonald et al, 2003, 2005a; Servan-Schreiber et al, 1996; Stratta et al, 1998). In the DPX task, dot patterns replace letters as stimuli, making it possible to more readily manipulate the visual similarity of stimuli and also reduce the influence of prior familiarity with the stimuli (MacDonald et al, 2005b), particularly useful for translational studies in monkeys and humans. In this study, we characterized performance on the DPX task before and after administering ketamine, an $N$-methyl-Daspartate (NMDA) receptor antagonist. There is considerable evidence that altered NMDA function may be a core component of the disease process producing schizophrenia (Coyle, 2012; Javitt and Zukin, 1991; Kantrowitz and Javitt, 2010; Lisman et al, 2008). Drugs that block neurotransmission at NMDA receptors are able to replicate the full spectrum of symptoms in schizophrenia, including positive, negative, and cognitive symptoms in human controls (Krystal et al, 1994). Here we provide the first evidence in monkeys that blocking neurotransmission at NMDA receptors replicates the specific error pattern that schizophrenia patients typically produce when performing the same context-processing task.

\section{MATERIALS AND METHODS}

\section{Subjects}

Two male rhesus macaques (Macaca mulatta), weighing $8.3-10.2 \mathrm{~kg}$, were subjects in this study. All animal care and experimental procedures conformed to National Institutes of Health guidelines and were in compliance with the protocols approved by the Animal Care and Use Committee at the University of Minnesota and Minneapolis Veterans Affairs Medical Center.

\section{Experimental Conditions}

Monkeys were seated in a primate chair in a soundattenuated, dimly illuminated room during task performance. Visual stimuli were varying spatial configurations of dots, which spanned 2.7-4.4 ${ }^{\circ}$ and were back-projected on a video screen $77.5 \mathrm{~cm}$ in front of the monkey by an LCD projector (Dell). Eye position was tracked using an infra-red eye tracking system (ISCAN, Woburn, MA), enabling the control of gaze strategies and visual input across conditions. During each trial until the response was made, monkeys were required to maintain visual fixation (within $2.7-3.9^{\circ}$ ) at the center of the screen marked by the fixation cross $\left(0.4^{\circ}\right)$. Failure to do so resulted in the trial aborting. Monkeys manipulated a joystick with their right hand in order to make a response during each trial.

\section{Task}

Monkeys were trained to perform the DPX task (Figure 1). Stimuli were various dot patterns that were comparable to those used in human studies (Jones et al, 2010). Each trial

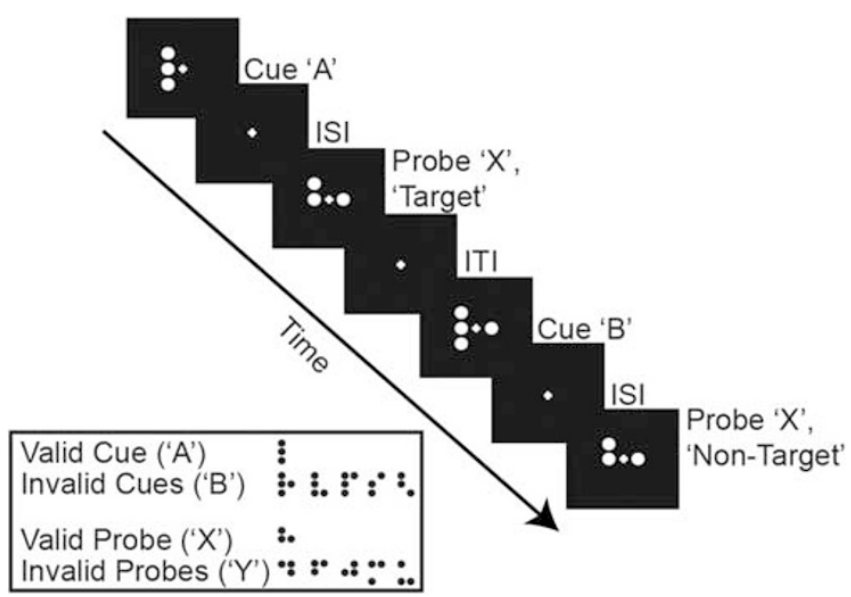

Figure I Schematic of the dot pattern expectancy (DPX) task. The dot patterns used as stimuli in the task are also depicted, grouped by their respective category designation. The monkeys performed the task while fixating at the cross at the center of the screen. 'Target' cue-probe sequences ('AX' trials) required a leftward motor response; 'non-target' cue-probe sequences (all other trial types) required a rightward motor response. ISI, inter-stimulus interval; ITI, intertrial interval. 
consisted of a sequence of two dot patterns, a cue stimulus (orange dots) followed by a probe stimulus (white dots) separated by a delay (Figure 1). The cue was presented for $1.0 \mathrm{~s}$. After a 1.0-s inter-stimulus delay, the second stimulus (probe) was presented for $0.5 \mathrm{~s}$. The intertrial interval was $1.1 \mathrm{~s}$. Monkeys had $1.5 \mathrm{~s}$ from the onset of the probe in which to respond. A valid cue (designated 'A') followed by a valid probe (designated ' $\mathrm{X}$ ') was the target sequence ('AX'). The correct response on target sequence trials was to move a joystick to the left after the probe appeared. A nontarget sequence was defined as one in which either the cue was invalid (collectively classified as ' $\mathrm{B}$ '), and/or the probe was invalid (collectively classified as ' $\mathrm{Y}$ '). Non-target trials consisted of 'AY', 'BX', or 'BY' sequences. The correct response on non-target trials was to move the joystick to the right. Monkeys were rewarded with sweetened water for correctly performed trials. Occasionally, to maintain a high level of baseline performance, additional rewards were given on a minority of trials in monkey 2 (Supplementary Methods). Directly before and following injection (saline or drug), monkeys performed at least 200 DPX trials (200-600 trial sets). Each set was a mixture of trial types with the following distribution: 69\% 'AX,' $12.5 \%$ 'AY', $12.5 \%$ 'BX', and $6 \%$ 'BY', the same proportions as used in human DPX studies with patients (Jones et al, 2010). Trials were selected for presentation in random order from each trial set. Monkeys were trained to perform at $\geqslant 80 \%$ accuracy on each trial type before testing began.

\section{Injection Regimen}

Monkeys received either a saline or ketamine injection intramuscularly into the hind limb each day following baseline trials. Post-injection trials began directly after the injection (within $5 \mathrm{~min}$ ). During baseline trials, each monkey had to perform at least 200 trials and achieve $\geqslant 80 \%$ accuracy on each trial type before an injection was given. Based on a pilot experiment in monkey 1, we determined that the $0.32-0.57 \mathrm{mg} / \mathrm{kg}$ dose range was optimal to test in the current experiment because in this range the monkey continued to work on the task but exhibited a substantial level of behavioral impairment. Four subanesthetic doses of ketamine $(0.32,0.40,0.49$, and $0.57 \mathrm{mg} / \mathrm{kg}$; diluted in saline) and saline alone were pseudo-randomly administered (0.28-0.34 ml injection volume) such that each monkey received two replications of each ketamine dose. A total of two saline and eight ketamine injections were given to monkey 1 while six saline and eight ketamine injections were given to monkey 2 .

\section{Data Analyses}

Behavioral performance in most cases had returned to near baseline by 40 -min post-injection of ketamine, so we restricted our analyses to the first $40 \mathrm{~min}$. On average postinjection, the monkeys completed 476 and 352 trials per day (monkey 1 and monkey 2, respectively) and these trials were used in the data analyses. Early response (monkey 1: $0.1 \%$ and monkey 2: $0.6 \%$ ), no response (monkey $1: 0.2 \%$ and monkey 2: $1.1 \%$ ), and fixation error (monkey $1: 12.8 \%$ and monkey 2: 17.8\%; Supplementary Figure S1, Supplementary Results) trials were excluded from data analysis. A $p<0.05$ was considered a statistically significant result for all data analyses.

In order to statistically evaluate the influence of ketamine on performance, we examined error rate data at the trial level using logistic regressions because the dependent variable in the analysis (trial success/failure) was a dichotomous variable. The independent variables in the model were injection status (pre- $v s$ post-injection) and dose (equation 1).

$$
\ln \left(\frac{p \text { (error })}{1-p(\text { error })}\right)=\beta_{0}+\beta_{1} \operatorname{Inj}+\beta_{2} \text { Dose }
$$

where $p$ (error) is the probability of an erroneous response, 'Inj' is the injection status, and 'Dose' is the ketamine dose. To examine differential effects of ketamine on the various trial types, we performed a logistic regression restricted to the post-injection behavioral data in which trial outcome was the dependent variable and trial type ('AX', 'BX', 'AY', or ' $\mathrm{BY}$ '), dose, and their interaction were independent variables (equation 2).

$$
\ln \left(\frac{p \text { (error })}{1-p(\text { error })}\right)=\beta_{0}+\beta_{1} \text { Type }+\beta_{2} \text { Dose }+\beta_{3} \text { Type } * \text { Dose }
$$

where 'Type' refers to the trial type. For the analysis of reaction time (RT) data, we used analysis of variance (ANOVA) followed by a Tukey-Kramer post hoc analysis on correct trials (Supplementary Methods).

The error rate data were further examined by computing $\mathrm{d}_{\text {context }}$ (Servan-Schreiber et al, 1996). The d' parameter is a sensitivity index that is defined as $Z$ (hit rate) $-Z$ (false alarm rate), where the function $Z(p)$ is the inverse of the cumulative Gaussian distribution. $\mathrm{D}_{\text {context }}$ is similar but is meant to measure specifically sensitivity to context in the AX-CPT and its variants (Servan-Schreiber et al, 1996). $\mathrm{D}^{\text {' }}$ context was computed as $\mathrm{Z}$ (proportion of correct ' $\mathrm{AX}$ ' trials) - Z(proportion of 'BX' errors) (Servan-Schreiber et al, 1996). Thus, as the proportion of ' $\mathrm{BX}$ ' errors (analogous to false alarms) approaches the proportion of ' $A X$ ' correct trials, $\mathrm{d}_{\text {' }}$ context will approach 0 indicating that monkeys tended to make the same response to the ' $\mathrm{X}$ ' cue regardless of whether the preceding cue was ' $A$ ' or ' $\mathrm{B}$ ', indicating low sensitivity to the cue and failure to use cue information to override habitual responding to the probe. A small constant correction of 0.001 was used to allow for estimation in the case of perfect accuracy (Servan-Schreiber et al, 1996). To confirm that changes in $\mathrm{d}_{\text {' }}$ context in response to ketamine did not simply reflect a directional response bias, we computed the difference between ' $B X$ ' and ' $A Y$ ' errors (MacDonald et al, 2003) for each replication of each ketamine dose. If there were high ' $B X$ ' errors and low 'AY' errors post drug this would indicate a problem in context processing and not a response bias (because incorrect responses on the 'BX' and 'AY' trial types are both to the left, and a response bias alone could therefore not account for a difference in these errors). In order to address whether deficits in context processing in the DPX task were due to deficits in early bottom-up visual processing, visualmotor association, or general visual attention to stimuli, we adapted $\mathrm{d}_{\text {context }}$ to examine sensitivity to the probe 
stimulus. We defined sensitivity to the probe as d' probe being equal to $\mathrm{Z}$ (proportion of correct 'AX' trials) $-\mathrm{Z}$ (proportion of 'AY' errors). We reasoned that any general deficit in visual processing or attention would equally affect sensitivity to both of the visual stimuli in the trial. Any greater loss of sensitivity to the cue relative to the probe after ketamine administration we therefore attributed to a context-processing deficit. The significance of the relation between dose and d' context (as well as 'BX' - 'AY' errors and $\mathrm{d}_{\text {probe }}$ ) was evaluated using linear regression.

Finally, we conducted logistic regression analyses to determine whether under ketamine, monkeys tended to commit perseverative 'BX' errors. (Perseveration analyses using the saline data were not performed because monkeys committed too few errors.) We evaluated whether, after making an error on a ' $\mathrm{BX}$ ' trial, the probability of making another error on the subsequent trial increased if that trial was a 'BX' trial relative to the other trial types. We restricted this analysis to the subset of trials in the data that followed a ' $\mathrm{BX}$ ' trial. We then used a logistic regression to evaluate whether success probability varied as a function of the trial type and whether the previous ' $\mathrm{BX}$ ' trial was performed correctly or not (equation 3; doses were collapsed for this analysis).

$$
\begin{aligned}
\ln \left(\frac{p(\text { error })}{1-p(\text { error })}\right)= & \beta_{0}+\beta_{1} \text { CurrentType }+\beta_{2} \text { PrevBXErr } \\
& +\beta_{3} \text { CurrentType } * \text { PrevBXErr }
\end{aligned}
$$

where 'Current Type' refers to the trial type of the current trial and 'PrevBXErr' refers to whether the previous ' $\mathrm{BX}$ ' trial was performed incorrectly. In addition, in order to examine the effect of dose on perseveration of the 'BX' trial type, we identified sequences of two consecutive 'BX' trials in the data. We then performed a logistic regression to contrast the rate of errors committed on the second ' $\mathrm{BX}$ ' trial as a function of dose and whether the previous ' $\mathrm{BX}$ ' trial was performed incorrectly (equation 4). Dose was entered into the logistic model as either low (0.32 and $0.40 \mathrm{mg} / \mathrm{kg})$ or high $(0.49$ and $0.57 \mathrm{mg} / \mathrm{kg})$, because too few 'BX' trial pairs were available to examine doses individually.

$$
\begin{aligned}
\ln \left(\frac{p(\text { error })}{1-p(\text { error })}\right)= & \beta_{0}+\beta_{1} \text { Dose }+\beta_{2} \text { PrevBXErr } \\
& +\beta_{3} \text { Dose } * \text { PrevBXErr }
\end{aligned}
$$

\section{RESULTS}

Error rate data were examined at the trial level. After ketamine administration, the monkeys' performance was worse as indicated by a significant increase in errors post-injection compared with baseline performances (monkey 1: Wald $_{1}=60.33, p<0.001$; monkey 2: Wald $_{1}=$ $29.43, p<0.001)$. This effect was dose dependent (monkey 1 : Wald $_{1}=76.48, p<0.001$; monkey 2 : $\mathrm{Wald}_{1}$ : 78.42, $p<0.001$ ) for both monkeys (Figures $2 \mathrm{a}$ and $\mathrm{b}$ ). The error rate remained low after saline (Figures $2 \mathrm{a}$ and $\mathrm{b}$, dose $0.0 \mathrm{mg} / \mathrm{kg}$; Figures $2 c$ and d, 'saline').

A more informative picture of the effects of ketamine on performances came from examining errors separated by trial type (Supplementary Table S1; Figures $2 \mathrm{c}$ and d). This allowed us to evaluate whether the effects of ketamine observed were more generalized or specific to trials with higher context-processing demands. A logistic model that included dose, trial type, and their interaction was significant for each factor in monkey 1 (dose: Wald $_{1}=$ 19.90, $p<0.001$; trial type: Wald $_{3}=10.47, p=0.015$; dose $\times$ trial type: $\left.\mathrm{Wald}_{3}=76.937, p<0.001\right)$. Trial type and the interaction between dose and trial type were significant in monkey 2 using the same model (trial type: $\mathrm{Wald}_{3}=39.49$, $p<0.001$; dose $\times$ trial type: Wald $\left._{3}=37.39, \quad p<0.001\right)$, although the main effect of dose was not. Dose was significant in monkey 2 when the interaction term was removed from the model (dose: $\mathrm{Wald}_{1}=88.28, p<0.001$ ).

As illustrated in Figures $2 c$ and $d$, most of the errors in both monkeys post-injection stemmed from poorer performance on the ' $\mathrm{BX}$ ' trial type. The ' $\mathrm{BX}$ ' trial type is the trial type that is most sensitive to patients' context-processing impairments (Figure 2e; Cohen et al, 1999; Jones et al, 2010; MacDonald et al, 2005b). By 40-min post-injection, there was no longer an increase in ' $\mathrm{BX}$ ' errors over the saline condition except at the highest dose in monkey 2 (Supplementary Figure S2; Supplementary Results).

Considering the effects on reaction time, ketamine produced a dose-dependent slowing of response that was most pronounced in monkey 2 (Figures $3 \mathrm{a}$ and $\mathrm{b}$ ). The pattern of the effect on RT over the different trial types was markedly different from the effect on percent correct performance (Supplementary Table S1, Figures 2 and 3). Although ketamine led to an increase in errors that was markedly more severe on ' $\mathrm{BX}$ ' trials relative to the other trial types, the effects of the drug on RT were less restricted to 'BX' trials and were more generalized across all the trial types (compare Figures $2 c$ and $d$ with Figures $3 c$ and $d$ ). Both animals exhibited a general pattern of slower responding on 'AY' and 'BY' trials relative to 'AX' and 'BX' trials that persisted across doses of ketamine in both animals (Figures $3 \mathrm{c}$ and $\mathrm{d}$ ). In an ANOVA applied to the RT data in monkey 1 , both main effects of dose $\left(F_{1}=244.64\right.$, $p<0.001)$, and trial type $\left(\mathrm{F}_{3}=336.69, p<0.001\right)$ were significant, as was the dose by trial type interaction $\left(\mathrm{F}_{3}=35.70\right.$, $p<0.001)$. In monkey 2 , there were also significant effects of dose $\left(\mathrm{F}_{1}=967.37, \quad p<0.001\right)$, trial type $\left(\mathrm{F}_{3}=66.90\right.$, $p<0.001)$, and dose by trial type interaction $\left(\mathrm{F}_{3}=31.76\right.$, $p<0.001)$. Monkeys were slowest to respond on 'AY' trials relative to the other trial types regardless of dose (TukeyKramer test; $p<0.05$ ), a result similar to that seen in humans (Jones et al, 2010). This provides evidence that in both species, suppressing the pre-potent target response to the probe after seeing an ' $A$ ' cue is a time-consuming process in the case that the probe is a ' $\mathrm{Y}$ '.

To further quantify the effect of ketamine on DPX performance we examined $\mathrm{d}_{\text {' }}$ context to measure the monkeys' sensitivity to context (the cue). Specificity to context was diminished with increasing dosage of ketamine in both monkeys (monkey 1: $\mathrm{F}_{1,8}=15.24, p=0.005$; Monkey 2: $\left.\mathrm{F}_{1,12}=71.94, p<0.001\right)$ such that $\mathrm{d}_{\text {context }}$ scores decreased with increasing dosage (Supplementary Table S1, Figure 4a). As these results may reflect a response bias, we examined another measure of context processing, the difference in 'BX' and 'AY' errors rates. A dose-dependent effect following drug was observed when differencing errors on 

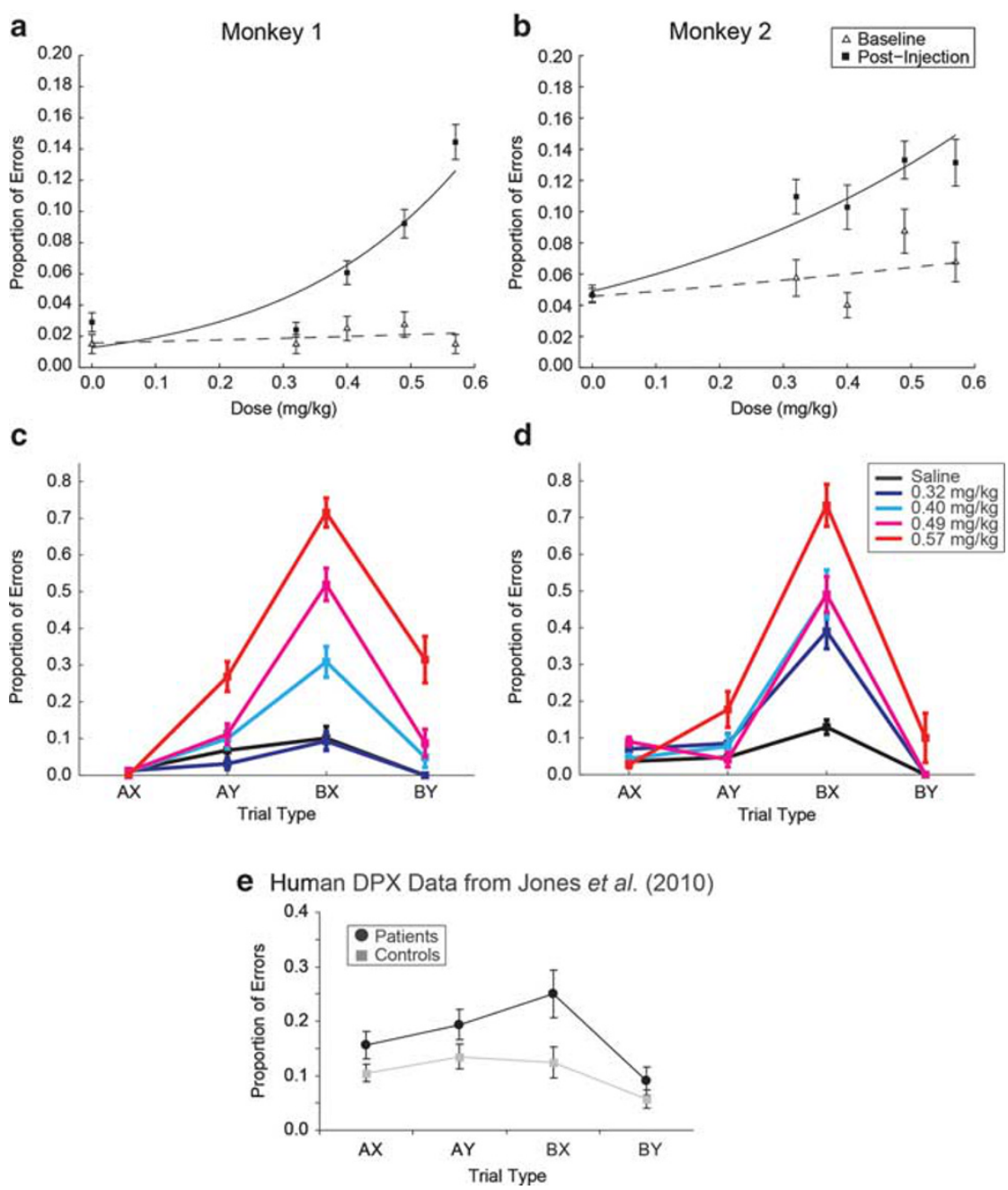

Figure 2 Effect of ketamine dose on performance accuracy (a-d). Replications for monkey I: two replications per drug dose and two replications of the saline condition. Replications for monkey 2: two replications per drug dose and six replications of the saline condition. (a, b) Overall performance accuracy by dose. Dashed line represents the logistic function fit line to the data for baseline performance. Solid line represents the logistic function fit to the data for post-injection performance. (c, d) Effect of ketamine dose on performance accuracy for each trial type. Error bars are I SEM. (e) Data illustrating schizophrenia patient $(n=47)$ vs control $(n=48)$ performance on the DPX task were adapted from a prior human study (Jones et al, 20 I 0). Copyright (C) 2010 by the American Psychological Association. The use of APA information does not imply endorsement by APA.

' $\mathrm{BX}$ ' and 'AY' trials (monkey 1: $\mathrm{F}_{1,8}=17.15, p=0.003$; monkey $\left.2: \mathrm{F}_{1,12}=35.71, p<0.001\right)$, such that with increasing dosage a higher proportion of errors on the 'BX' trial type versus 'AY' was observed (Figure $4 \mathrm{~b}$ ). These results complement the $\mathrm{d}_{\text {context }}$ results indicating ketamine induced a context-processing deficit that could not simply be accounted for by a response bias.

In order to examine whether ketamine induced deficits in the DPX task (Figures 2 and 4) that could be attributed to deficits in visual processing, visual-motor association, or general visual attention to stimuli, we measured sensitivity to the probe using $d^{\prime}$ probe. Ketamine-induced sensory motor or attention deficits should equally impact processing of the cue and the probe. Therefore, any greater impact of ketamine on cue processing could be attributed to context processing. We found that, in contrast to sensitivity to the cue, sensitivity to the probe was not diminished by increasing doses of ketamine in both monkeys (monkey 1: $\mathrm{F}_{1,8}=0.063, p=0.808 ;$ monkey $2: \mathrm{F}_{1,12}=4.05, p=0.067$ ) such that $d$ 'probe scores were not significantly different among doses examined (Figure 4c). Monkeys did exhibit an increased probability to break fixation under ketamine (Supplementary Figure S1; Supplementary Results). However, this effect did not account for the increase in ' $\mathrm{BX}$ ' errors (Figure 2) because fixation errors were excluded from the analysis of the behavioral data. Another possible account of the ketamine effect we observed is that it disrupted the ability to switch between alternative response rules in the task. Considering ' $\mathrm{X}$ ' probe trials, the cue (' $\mathrm{A}$ ' or ' $\mathrm{B}$ ') dictated which of two competing responses to the probe was correct. However, we found that ' $\mathrm{BX}$ ' errors were no more frequent on cue-switch than cue-repeat trials (Supplementary Figure S3, Supplementary Results).

Finally, the high number of trials collected allowed us to examine a novel aspect of context processing, namely perseveration of ' $\mathrm{BX}$ ' errors in the ketamine condition. Perseveration of errors is often seen in schizophrenia patients where patients continue to make the same mistakes 

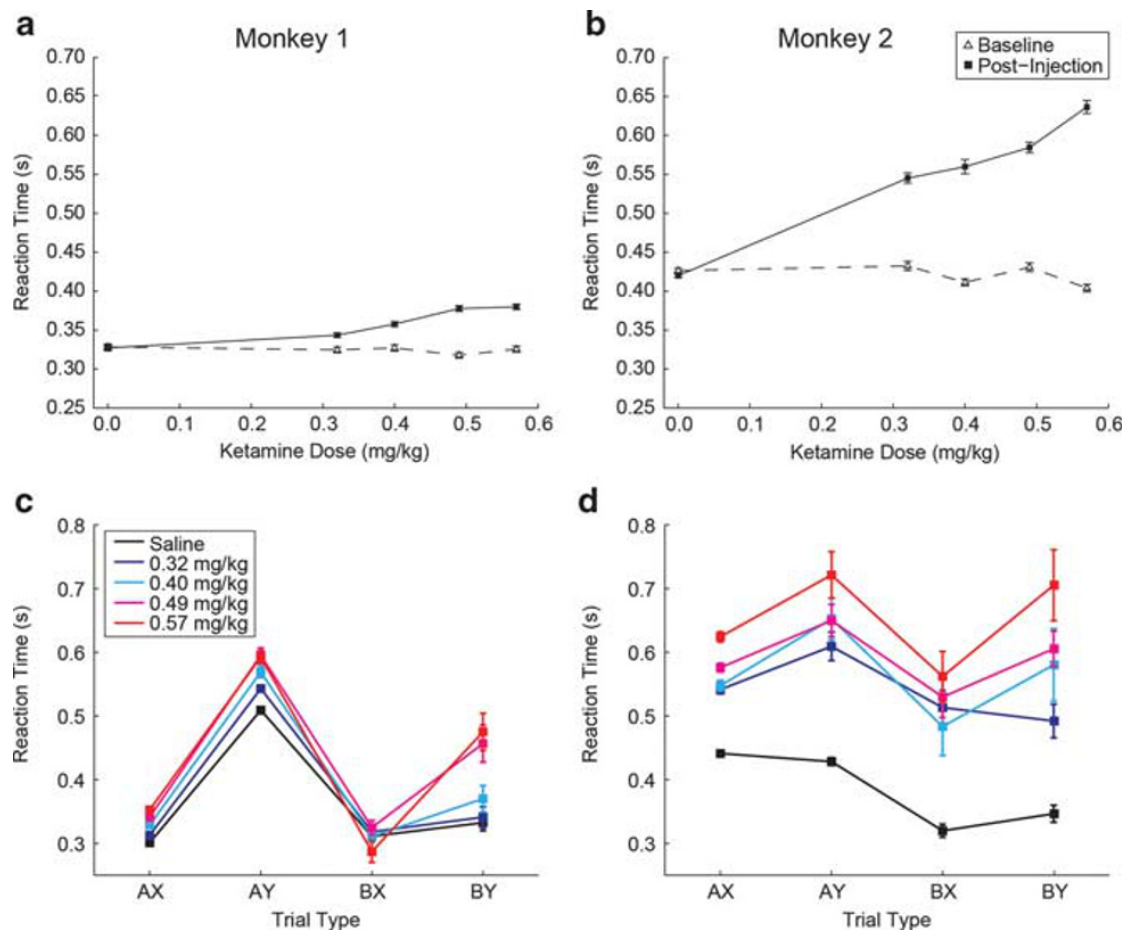

Figure 3 Effect of ketamine dose on reaction time. Replications for monkey I: two replications per drug dose and two replications of the saline condition. Replications for monkey 2: two replications per drug dose and six replications of the saline condition. (a, b) Overall reaction time by dose. Dashed line represents baseline performance. Solid line represents post-injection performance. (c, d) Effect of ketamine dose on reaction time for each trial type. Error bars $=\mathrm{SEM}$.

repeatedly over consecutive trials and are seemingly ignoring information instructing them to change their strategy (Waford and Lewine, 2010). To examine whether monkeys were perseverating on the DPX task, we evaluated errors on trials that were preceded by 'BX' trials (the trial type with the greatest number of errors to examine). Following a 'BX' error, the probability that monkeys would commit another error was markedly higher if the subsequent trial was also a ' $\mathrm{BX}$ ' trial relative to the other trial types (Figures 5a and b). A logistic model that included current trial type, previous 'BX' error, and their interaction was significant for the interaction term in both monkeys (monkey 1: Wald $_{3}=16.649, \quad p=0.001$; monkey 2: Wald $\left._{3}=10.422, p=0.015\right)$. As the two monkeys exhibited the same general pattern in response to ketamine (Figures $5 \mathrm{a}$ and $\mathrm{b}$ ) and there were relatively few consecutive ' $\mathrm{BX}$ ' trials in the data, we pooled the data across the two animals to examine whether ketamine effects on 'BX' perseveration were dose dependent. We found that ketamine increased perseveration on 'BX' trials in a dose-dependent manner (Figures $5 c$ and d; dose: Wald $_{1}=10.27, p=0.001$; previous error: Wald $_{1}=15.37, \quad p<0.001$; dose $\times$ previous error: Wald $_{1}=4.24, p<0.039$ ), suggesting that ketamine administration affected the use of feedback to increase contextprocessing resources. We further examined whether ' $\mathrm{BX}$ ' errors under ketamine could be entirely attributed to perseveration, and found evidence to the contrary. Specifically, in addition to increasing perseverative errors, ketamine increased nonperseverative 'BX' errors as well (defining nonperseverative errors as errors that followed successfully performed trials). The increase in nonperseverative errors was dose dependent in both monkeys (Figure 5e; Supplementary Results).

\section{DISCUSSION}

In this study, we provide the first evidence that NMDA receptor blockade in monkeys can replicate the contextprocessing deficit that has been thoroughly characterized in the human schizophrenia patient population (Barch et al, 2003; Chung et al, 2011; Cohen et al, 1999; Henderson et al, 2012; Jones et al, 2010; Lopez-Garcia et al, 2012; MacDonald et al, 2003, 2005a, b; Servan-Schreiber et al, 1996; Stratta et al, 1998; Zhang et al, 2012) and replicated in human controls given ketamine (Umbricht et al, 2000). Results were robust and specific in both animals, with monkey 1 (Figure 2c) and monkey 2 (Figure 2d) exhibiting a highly similar pattern of errors over trial types under ketamine. The consistency of the results across animals provides compelling evidence that NMDA antagonists in monkeys can replicate context-processing deficits seen in schizophrenia patients. Future studies will be needed to evaluate the question of individual variability in these deficits and what genetic and/or environmental factors might contribute to them. Previous studies have shown that NMDA antagonists impair working memory function in monkeys (Boyce et al, 1991; Roberts et al, 2010a, b; Taffe et al, 2002; Tsukada et al, 2005), modeling one important aspect of cognitive dysfunction in schizophrenia (Castner et al, 2004; 
Park and Holzman, 1992). However, our data extend and refine prior efforts to translate cognitive deficits in schizophrenia to monkeys in several key respects.

First, by showing that ketamine can replicate contextprocessing deficits we broaden the scope of cognitive

a


dysfunction in schizophrenia that has been effectively replicated in an animal model. In the case that contextual cue and imperative probe stimuli are separated by a delay, context processing incorporates working memory. However, context-processing tasks measure processes that extend beyond the domain of working memory to incorporate the additional element of executive control (Cohen et al, 1999; MacDonald, 2008). For example, attenuated impairment in context processing is observed in patients performing the AX-CPT when there is no working memory requirement (MacDonald et al, 2003), indicating that context-processing deficits involve disruption of processes that are not restricted to working memory alone. We found that monkeys given ketamine (Figures $2 \mathrm{c}$ and d), just like patients with schizophrenia (Figure 2e; MacDonald, 2008), are selectively impaired in the cognitive and behavioral flexibility that the DPX task requires and which is a fundamental component of cognitive deficits in the disease. The one prior study in which monkeys were trained to perform a variant of the AX-CPT (Dias et al, 2006) did not examine the effects of NMDA receptor blockade on performance, which was the focus of this study. The specific increase in 'BX' errors after ketamine administration we observed in monkeys performing the DPX task could not be attributed to disruption of other processes such as rule switching or be entirely explained by an increase in perseveration, adding to the evidence that errors in the task reflected a failure of context processing (Figure 5e and Supplementary Figure S3, Supplementary Results).

Second, we trained monkeys to perform the same behavioral paradigm used to evaluate cognitive function in human patients with schizophrenia, equating the aspects of the task (such as the type of visual stimuli and overall task complexity) that could greatly complicate quantitative comparisons of behavioral performance patterns between the two species. Many prior studies examining the same cognitive functions in humans and monkeys have used substantially different paradigms to measure the cognitive function of interest. For example, working memory processes evaluated in monkeys with spatial delayed response tasks that require monkeys to retrieve a bait item from a covered well after a delay (Roberts et al, 2010a,b), could differ from working memory processes in humans evaluated with n-back and span tasks, which present different stimuli, require different motor responses, and are substantially more complex (Lee and Park, 2005).

Third, one of the greatest potential confounds in neuropsychological testing of cognitive dysfunction in

Figure 4 Effect of ketamine dosage on context (a, b) and probe processing (c). Dashed (monkey I) and solid (monkey 2 ) lines represent linear fits obtained by regressing performance data onto ketamine dose. Replications for monkey I: two replications per drug dose and two replications of the saline condition. Replications for monkey 2: two replications per drug dose and six replications of the saline condition.

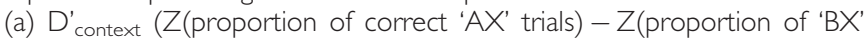
errors)) values for each replication of each dose in the experiment. (b) Difference in proportion of 'BX' and 'AY' errors for each replication of each dose in the experiment. (c) D' probe ( $Z$ (proportion of correct 'AX' trials) $-Z$ (proportion of 'AY' errors)) values for each day of the experiment. 

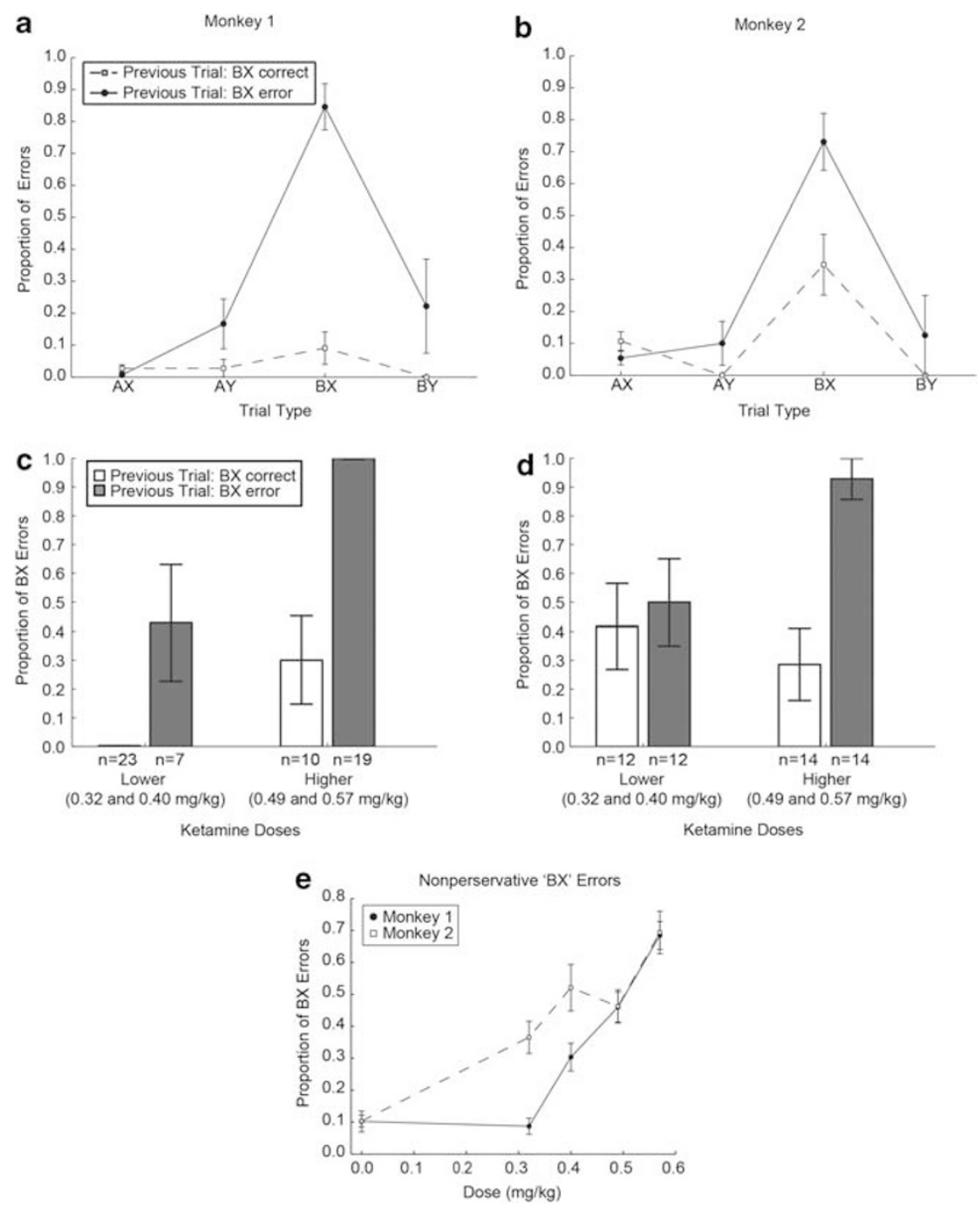

Figure 5 Proportion of perseverative 'BX' errors after ketamine administration (a-d). Replications for monkey I: two replications per drug dose and two replications of the saline condition. Replications for monkey 2: two replications per drug dose and six replications of the saline condition. (a, b) Performance accuracy on trials that followed 'BX' trials during the experiment, as a function of trial type and previous 'BX' trial performance (data collapsed across dose). $(c, d)$ Effect of ketamine dose on 'BX' perseverative errors. Analysis restricted to consecutive pairs of 'BX' trials during the experiment. Bars plot the proportion of erroneous trials on the second 'BX' trial as a function of whether the monkey performed correctly (open bars) or incorrectly (filled bars) on the previous 'BX' trial. (e) Effect of ketamine dose on nonperseverative 'BX' errors. Proportion of 'BX' errors on 'BX' trials that followed a correctly performed trial as a function of ketamine dose. Error bars = SEM.

schizophrenia is the possibility that behavioral paradigms measure a generalized deficit in the disease rather than an impairment in a specific cognitive process (MacDonald, 2008). Our data provide strong evidence that ketamine in monkeys produces a specific cognitive deficit in context processing that can be dissociated from a generalized deficit in more global processes such as vigilance, attention, motivation (Figures $2 \mathrm{c}$ and d, Figures $4 \mathrm{a}$ and b), general stimulus processing (Figure 4c), or motor response bias (Figure 4b). Our behavioral results could reflect a deficit in top-down control of visual processing that selectively impaired encoding of the cue stimulus as has been previously proposed (Dias et al, 2011). However, to our view, a deficit in visual processing that selectively disrupts visual processing of contextual cues could be considered to constitute a deficit in one aspect of context processing.

Fourth, the context-processing task we have adopted for this translational study incorporates four distinct trial conditions in the task design. This provides two advantages: it significantly increases the behavioral resolution with which we are able to compare performance patterns across the two species, and it further adds to our ability to differentiate a selective impairment in context processing from a more generalized cognitive impairment (Supplementary Discussion). Both monkeys given ketamine (Figures 2c and d) and patients with schizophrenia (Figure 2e) performing the DPX task exhibit a selective and dramatic increase in errors on 'BX' trials, and are relatively unimpaired on the remaining trial types. Humans given ketamine (Umbricht et al, 2000) and some schizophrenia patients performing the AX-CPT (Cohen et al, 1999) also demonstrate greater 'AX' errors; a result not observed in this study. Monkeys had much greater experience performing the task than human subjects (extensive training was necessary given the complexity of the task), which may have resulted in the 'AX' performance difference since monkeys 
had a longer time to develop habitual responding to 'target' trials.

Fifth, our data provide a particularly direct translation of executive control impairments in schizophrenia to monkeys. One prior study has shown that ketamine administered to two monkeys performing an executive control task impaired their ability to select between competing responses to the same stimulus instructed by alternative rules, an effect known as congruity cost (Stoet and Snyder, 2006). However, it is not readily feasible on the basis of these prior data to directly compare executive control deficits in monkeys given ketamine and schizophrenia patients because the task used to index this function in that prior study in monkeys (Stoet and Snyder, 2006) has not yet been tested in human patients with schizophrenia. Congruity costs have been observed in a related executive control task in schizophrenia patients (Wylie et al, 2010). However, that task involved letter identification, a condition not tested in monkeys (Stoet and Snyder, 2006).

Sixth, ours is the first study to show that ketamine increases perseverative errors in context-processing tasks (Figures 5a-d). This increases confidence in the translational validity of our nonhuman primate model, as perseverative errors are a hallmark of patient performance in other executive control tasks (Perry and Braff, 1998; Waford and Lewine, 2010).

In summary, our data demonstrate that administration of an NMDA receptor antagonist to monkeys performing the DPX task can replicate a schizophrenia-like performance pattern that is indicative of a selective deficit in context processing. Our data suggest that knocking down NMDA synaptic function is sufficient to produce context-processing deficits. They do not provide evidence that NMDA hypofunction is necessary, however, and we cannot rule out the possibility that these cognitive deficits stem from dysfunction at other receptors in patients or that there are multiple neurotransmitter systems involved. For example, human control subjects given the $5-\mathrm{HT}_{2 \mathrm{~A}}$ agonist psilocybin also demonstrate context-processing deficits (Umbricht et al, 2003). In addition, the $\alpha_{2 \mathrm{~A}}$ receptor agonist gaunfacine has been shown in one study to ameliorate contextprocessing dysfunction in schizotypal personality disorder patients (McClure et al, 2007). These data suggest that multiple transmitter systems independently converge on the neural systems that normally mediate context processing, or that these transmitter systems are functionally coupled. By the same token, our data indicating that blocking NMDA receptors disrupts context processing suggests that treatments that augment the NMDA synaptic function may be beneficial in treating context-processing dysfunction in schizophrenia. Glycine is a co-agonist of the NMDA receptor and administration of glycine transport inhibitors has been shown to restore of working memory in monkeys given ketamine (Roberts et al, 2010b). Glycine transport inhibitors are currently in different phases of clinical trials in humans to test their efficacy in the patient population (Javitt, 2012), although it is not yet known whether these compounds will prove effective in reversing deficits in context processing in schizophrenia. To our knowledge, it has not yet been tested whether drugs that act at other synapses are able to induce context-processing deficits in animals.
As cognitive function predicts functional capacity in the patient population (Green et al, 2000), it is essential to develop treatments that effectively address cognitive dysfunction in the disease. Context processing does not account for the full spectrum of cognitive dysfunction in schizophrenia and restoration of this process alone is not likely to restore normal social and cognitive function in patients entirely. However, patients' performance on the DPX task is positively correlated with general levels of functioning (Gold et al, 2012). In addition, the DPX task isolates a relatively circumscribed and specific cognitive process (context processing), perhaps to a greater degree than more traditional neuropsychological tests (Gold et al, 2012). This could narrow the search for the causal neural mechanisms that underlie cognitive deficits in the disease. As it is often easier to solve a more clearly delineated problem, targeting transmitter systems and neural mechanisms that enable context processing may therefore have significant utility in early drug development. Now that context-processing dysfunction on the DPX task has been demonstrated in monkeys given ketamine, this model could facilitate discovery of more effective ways to restore normal cognitive function in schizophrenia.

\section{FUNDING AND DISCLOSURE}

Rachael Blackman has received funding to support the work contained in this manuscript as outlined in the Acknowledgements section of this manuscript. Rachael Blackman has received funding from the University of Minnesota and U.S. National Institutes of Health. Dr MacDonald receives funding through the U.S. National Institutes of Health. Dr Chafee receives funding through the U.S. National Institutes of Health.

\section{ACKNOWLEDGEMENTS}

This work was supported by the National Institutes of Health (R01 MH077779), the Department of Veterans Affairs, and the American Brain Sciences Chair. In addition, Rachael Blackman was supported by NIH MSTP grant T32 GM008244 and the Wilfred Wetzel Graduate Fellowship. We thank Dr David Crowe for important insights into the data analyses for this study as well as valuable discussions regarding the manuscript. In addition, we thank Dean Evans and Dale Boeff for their exceptional technical support of this work (animal care and computer programming, respectively). The views and opinions expressed in this paper are those of the authors and not those of the United States Federal Government.

\section{REFERENCES}

Abramczyk RR, Jordan DE, Hegel M (1983). "Reverse" stroop effect in the performance of schizophrenics. Perceptual and motor skills 56: 99-106.

Barch DM, Carter CS, MacDonald AW 3rd, Braver TS, Cohen JD (2003). Context-processing deficits in schizophrenia: diagnostic specificity, 4-week course, and relationships to clinical symptoms. J Abnorm Psychol 112: 132-143. 
Barch DM, Mitropoulou V, Harvey PD, New AS, Silverman JM, Siever LJ (2004). Context-processing deficits in schizotypal personality disorder. J Abnorm Psychol 113: 556-568.

Boyce S, Rupniak NM, Steventon MJ, Cook G, Iversen SD (1991). Psychomotor activity and cognitive disruption attributable to NMDA, but not sigma, interactions in primates. Behav Brain Res 42: 115-121.

Brambilla P, Macdonald AW 3rd, Sassi RB, Johnson MK, Mallinger AG, Carter CS et al (2007). Context processing performance in bipolar disorder patients. Bipolar disorders 9: 230-237.

Castner SA, Goldman-Rakic PS, Williams GV (2004). Animal models of working memory: insights for targeting cognitive dysfunction in schizophrenia. Psychopharmacology (Berl) 174: $111-125$.

Chung YS, Mathews JR, Barch DM (2011). The effect of context processing on different aspects of social cognition in schizophrenia. Schizophr Bull 37: 1048-1056.

Cohen JD, Barch DM, Carter C, Servan-Schreiber D (1999). Context-processing deficits in schizophrenia: converging evidence from three theoretically motivated cognitive tasks. J Abnorm Psychol 108: 120-133.

Cohen JD, Servan-Schreiber D (1992). Context, cortex, and dopamine: a connectionist approach to behavior and biology in schizophrenia. Psychol Rev 99: 45-77.

Coyle JT (2012). NMDA receptor and schizophrenia: a brief history. Schizophr Bull 38: 920-926.

Dias EC, Butler PD, Hoptman MJ, Javitt DC (2011). Early sensory contributions to contextual encoding deficits in schizophrenia. Arch Gen Psychiatry 68: 654-664.

Dias EC, McGinnis T, Smiley JF, Foxe JJ, Schroeder CE, Javitt DC (2006). Changing plans: neural correlates of executive control in monkey and human frontal cortex. Exp Brain Res 174: 279-291.

Gold JM, Barch DM, Carter CS, Dakin S, Luck SJ, MacDonald AW 3rd et al (2012). Clinical, functional, and intertask correlations of measures developed by the cognitive neuroscience test reliability and clinical applications for schizophrenia consortium. Schizophr Bull 38: 144-152.

Goodwin SJ, Blackman RK, Sakellaridi S, Chafee MV (2012). Executive control over cognition: stronger and earlier rule-based modulation of spatial category signals in prefrontal cortex relative to parietal cortex. J Neurosci 32: 3499-3515.

Green MF, Kern RS, Braff DL, Mintz J (2000). Neurocognitive deficits and functional outcome in schizophrenia: are we measuring the "right stuff"? Schizophr Bull 26: 119-136.

Henderson D, Poppe AB, Barch DM, Carter CS, Gold JM, Ragland JD et al (2012). Optimization of a goal maintenance task for use in clinical applications. Schizophr Bull 38: 104-113.

Holmes AJ, MacDonald A 3rd, Carter CS, Barch DM, Andrew Stenger V, Cohen JD (2005). Prefrontal functioning during context processing in schizophrenia and major depression: an event-related fMRI study. Schizophr Res 76: 199-206.

Javitt DC (2012). Glycine transport inhibitors in the treatment of schizophrenia. Handbook Experimental Pharmacol 213: 367-399.

Javitt DC, Zukin SR (1991). Recent advances in the phencyclidine model of schizophrenia. Am J Psychiatry 148: 1301-1308.

Jones JA, Sponheim SR, MacDonald AW 3rd (2010). The dot pattern expectancy task: reliability and replication of deficits in schizophrenia. Psychol Assess 22: 131-141.

Kantrowitz JT, Javitt DC (2010). N-methyl-D-aspartate (NMDA) receptor dysfunction or dysregulation: the final common pathway on the road to schizophrenia? Brain Res Bull 83: $108-121$.

Krystal JH, Karper LP, Seibyl JP, Freeman GK, Delaney R, Bremner JD et al (1994). Subanesthetic effects of the noncompetitive NMDA antagonist, ketamine, in humans. Psychotomimetic, perceptual, cognitive, and neuroendocrine responses. Arch Gen Psychiatry 51: 199-214.
Lee J, Park S (2005). Working memory impairments in schizophrenia: a meta-analysis. J Abnorm Psychol 114: 599-611.

Lisman JE, Coyle JT, Green RW, Javitt DC, Benes FM, Heckers S et al (2008). Circuit-based framework for understanding neurotransmitter and risk gene interactions in schizophrenia. Trends Neurosci 31: 234-242.

Lopez-Garcia P, Young Espinoza L, Molero Santos P, Marin J, Ortuno Sanchez-Pedreno F (2012). Impact of COMT genotype on cognition in schizophrenia spectrum patients and their relatives. Psychiatry Res (http://dx.doi.org/10.1016/j.psychres. 2012.09.043).

MacDonald AW 3rd (2008). Building a clinically relevant cognitive task: case study of the AX paradigm. Schizophr Bull 34: 619-628.

MacDonald AW 3rd, Carter CS, Kerns JG, Ursu S, Barch DM, Holmes AJ et al (2005a). Specificity of prefrontal dysfunction and context processing deficits to schizophrenia in nevermedicated patients with first-episode psychosis. Am J Psychiatry 162: 475-484

MacDonald AW 3rd, Goghari VM, Hicks BM, Flory JD, Carter CS, Manuck SB (2005b). A convergent-divergent approach to context processing, general intellectual functioning, and the genetic liability to schizophrenia. Neuropsychology 19: 814-821.

MacDonald AW 3rd, Pogue-Geile MF, Johnson MK, Carter CS (2003). A specific deficit in context processing in the unaffected siblings of patients with schizophrenia. Arch Gen Psychiatry 60: 57-65.

McClure MM, Barch DM, Romero MJ, Minzenberg MJ, Triebwasser J, Harvey PD et al (2007). The effects of guanfacine on context processing abnormalities in schizotypal personality disorder. Biol Psychiatry 61: 1157-1160.

Miller EK, Cohen JD (2001). An integrative theory of prefrontal cortex function. Annu Rev Neurosci 24: 167-202.

Mortimer AM (1997). Cognitive function in schizophrenia-do neuroleptics make a difference? Pharmacol Biochem Behav 56: 789-795.

Nuechterlein KH, Dawson ME (1984). Information processing and attentional functioning in the developmental course of schizophrenic disorders. Schizophr Bull 10: 160-203.

Park S, Holzman PS (1992). Schizophrenics show spatial working memory deficits. Arch Gen Psychiatry 49: 975-982.

Perry W, Braff DL (1998). A multimethod approach to assessing perseverations in schizophrenia patients. Schizophr Res 33: 69-77.

Roberts BM, Seymour PA, Schmidt CJ, Williams GV, Castner SA (2010a). Amelioration of ketamine-induced working memory deficits by dopamine D1 receptor agonists. Psychopharmacology (Berl) 210: 407-418.

Roberts BM, Shaffer CL, Seymour PA, Schmidt CJ, Williams GV, Castner SA (2010b). Glycine transporter inhibition reverses ketamine-induced working memory deficits. Neuroreport 21: 390-394.

Servan-Schreiber D, Cohen JD, Steingard S (1996). Schizophrenic deficits in the processing of context. A test of a theoretical model. Arch Gen Psychiatry 53: 1105-1112.

Stip E, Chouinard S, Boulay LJ (2005). On the trail of a cognitive enhancer for the treatment of schizophrenia. Prog Neuropsychopharmacol Biol Psychiatry 29: 219-232.

Stoet G, Snyder LH (2006). Effects of the NMDA antagonist ketamine on task-switching performance: evidence for specific impairments of executive control. Neuropsychopharmacology 31: $1675-1681$.

Stratta P, Daneluzzo E, Bustini M, Casacchia M, Rossi A (1998). Schizophrenic deficits in the processing of context. Arch Gen Psychiatry 55: 186-188.

Sullivan EV, Mathalon DH, Zipursky RB, Kersteen-Tucker Z, Knight RT, Pfefferbaum A (1993). Factors of the Wisconsin Card Sorting Test as measures of frontal-lobe function in schizophrenia and in chronic alcoholism. Psychiatry research 46: 175-199. 
Taffe MA, Davis SA, Gutierrez T, Gold LH (2002). Ketamine impairs multiple cognitive domains in rhesus monkeys. Drug Alcohol Depend 68: 175-187.

Tsukada H, Nishiyama S, Fukumoto D, Sato K, Kakiuchi T, Domino EF (2005). Chronic NMDA antagonism impairs working memory, decreases extracellular dopamine, and increases D1 receptor binding in prefrontal cortex of conscious monkeys. Neuropsychopharmacology 30: 1861-1869.

Umbricht D, Schmid L, Koller R, Vollenweider FX, Hell D, Javitt DC (2000). Ketamine-induced deficits in auditory and visual context-dependent processing in healthy volunteers: implications for models of cognitive deficits in schizophrenia. Arch Gen Psychiatry 57: 1139-1147.

Umbricht D, Vollenweider FX, Schmid L, Grubel C, Skrabo A, Huber T et al (2003). Effects of the 5-HT2A agonist psilocybin on mismatch negativity generation and $\mathrm{AX}$-continuous performance task: implications for the neuropharmacology of cognitive deficits in schizophrenia. Neuropsychopharmacology 28: 170-181.

Waford RN, Lewine R (2010). Is perseveration uniquely characteristic of schizophrenia? Schizophr Res 118: 128-133.

Wylie GR, Clark EA, Butler PD, Javitt DC (2010). Schizophrenia patients show task switching deficits consistent with N-methyld-aspartate system dysfunction but not global executive deficits: implications for pathophysiology of executive dysfunction in schizophrenia. Schizophr Bull 36: 585-594.

Zhang Q, Shen Q, Xu Z, Chen M, Cheng L, Zhai J et al (2012). The effects of CACNA1C gene polymorphism on spatial working memory in both healthy controls and patients with schizophrenia or bipolar disorder. Neuropsychopharmacology 37: 677-684.

Supplementary Information accompanies the paper on the Neuropsychopharmacology website (http://www.nature.com/npp) 